\title{
A comparative study on the effect of docetaxel-albumin nanoparticles and docetaxel-loaded PEG-albumin nanoparticles against non-small cell lung cancer
}

\author{
GUANGMING JIN $^{1,2}$, MINGJI JIN ${ }^{1}$, XUEZHE YIN ${ }^{2}$, ZHEHU JIN $^{1}$, LIQING CHEN ${ }^{1}$ and ZHONGGAO GAO ${ }^{1}$ \\ ${ }^{1}$ State Key Laboratory of Bioactive Substance and Functions of Natural Medicines, Beijing Key Laboratory \\ of Drug Delivery Technology and Novel Formulations, Chinese Academy of Medical Sciences and Peking \\ Union Medical College, Beijing 100050; ${ }^{2}$ Yanbian University Hospital, Yanji, Jilin 133000, P.R. China
}

Received July 25, 2015; Accepted September 9, 2015

DOI: 10.3892/ijo.2015.3174

\begin{abstract}
The present study mainly compared the effect of docetaxel-albumin nanoparticles (DANPs) and docetaxelloaded PEG-albumin nanoparticles (PEG-DANPs) against non-small cell lung cancer (NSCLC). We made systematic assessments on these three drugs against NSCLC both in vitro and in vivo. With the purpose of eliminating side-effects of the commercial formulation (Tween-80) and prolonging the blood circulation time, we used emulsion-evaporation crosslink method to prepare DANPs and PEG-DANPs. The DANPs had an average particle size of $163.4 \pm 3.76 \mathrm{~nm}$, a zeta potential of $-19.4 \pm 0.18 \mathrm{mV}$, a polydispersity index of $0.143 \pm 0.03$, a drug loading of $8.71 \pm 0.98 \%$, and an encapsulation efficiency of $93.58 \pm 0.86 \%$; the average particle size of PEG-DANPs is $169.19 \pm 2.36 \mathrm{~nm}$, zeta potential is $-18.2 \pm 0.21 \mathrm{mV}$, with a polydispersity index of $1.56 \pm 0.05$, a drug loading of $8.72 \pm 1.05 \%$ and an encapsulation efficiency of $95.4 \pm 5.5 \%$. PEG-DANPs showed a dose- and time-dependent efficacy in cytotoxicity studies in vitro; the hemolysis test indicated that PEG-DANPs had less hemocytolysis than Aisu ${ }^{\circledR}$ and DANPs; in addition, a more prolonged circulation time and sustained in vitro release behavior were observed in the PEG-DANPs compared with Aisu ${ }^{\circledR}$ and DANPs; the cellular uptake test in vitro demonstrated that PEG-DANPs could be absorbed easier into the nucleus; furthermore, the tumor growth of NSCLC-bearing nude mice in vivo was reduced the most by PEG-DANPs. In conclusion, the PEG-DANPs have the lowest side-effects, the highest antitumor activity with the
\end{abstract}

Correspondence to: Professor Zhonggao Gao, State Key Laboratory of Bioactive Substance and Functions of Natural Medicines, Beijing Key Laboratory of Drug Delivery Technology and Novel Formulations, Chinese Academy of Medical Sciences and Peking Union Medical College, Beijing 100050, P.R. China E-mail: zggao@imm.ac.cn

Key words: non-small cell lung cancer, A549, docetaxel, albumin nanoparticles, PEG longest blood circulation time of the three drugs, and it will provide an alternative to patients with NSCLC.

\section{Introduction}

Lung cancer is a very common malignant tumor with incidence and mortality rates the first worldwide, almost one million patients are diagnosed with lung cancer each year all over the world. NSCLC accounts for $75-85 \%$ among the total number of lung cancer, and elderly patients aged $>65$ years accounted for $>50 \%$ (1). It is always at the late stage when patients were discovered (stage III or IV) and the 5-year survival rate is only $12-15 \%(2,3)$, thus, these patients cannot receive surgical treatment $(4,5)$.

For elderly advanced NSCLC patients, chemotherapy is still the first choice of treatment $(6,7)$, it is a relatively effective treatment for elderly NSCLC patients who cannot tolerant surgical operation. It can effectively reduce the progress of lung cancer and the recurrence, enhance the effect of clinical treatment, prolong patient survival rate and improve their quality of life (8).

There are some chemotherapy regimens and drugs for elderly NSCLC patients, such as cisplatin, VP-16, gemcitabine, paclitaxel and docetaxel (9). However, there is lack of a mature and effective way for NSCLC because most of the elderly patients refuse or are unable to complete the treatment. So how to choose a drug with good effect and little side reaction is a hot spot in tumor research $(10,11)$. Docetaxel is a cell cycle specific antitumor drug which is applied to cells at the $\mathrm{M}$ phase. It is a relatively new type of anti-microtubule taxane drug, and it is the only one approved for first- and second-line chemotherapy for NSCLC drug treatment by the U.S. Food and Drug Administration (FDA) and the EU. According to the existing clinical data of current studies, DTX has been proved very effective for NSCLC $(12,13)$.

However, the low solubility of DTX severely limits its clinical application. Polysorbate 80 (Tween-80) in saline solution was used to enhance the solubility of DTX in commercial products, such as Taxotere ${ }^{\circledR}$ and Aisu ${ }^{\circledR}$, it can induce hemolysis reaction and allergic reaction which is very common 
in clinical use. DTX in these two commercial products (Taxotere and Aisu) showed non-specific toxicities to normal organs, which led to intolerable side-effects such as peripheral neuropathy, hemolytic reaction and hypersensitivity reactions (14). Furthermore, the short elimination half-life of DTX in commercial products cause to more frequent injection in clinic which increase inconvenience and pain for the patients (15). Therefore, removing the side-effects and improving the half-life of DTX is getting ihncreased attention (16).

Biodegradable albumin nanoparticles have received attention in cancer targeted therapeutics during the past few decades (17). Previous reports have proven that drug-loaded polymeric nanoparticles could accumulate in certain tumors more efficiently than other carriers by enhancing permeability and retention (EPR) effect $(18,19)$. Another advantage is the long circulating half-life and lower systemic toxicity which is superior to conventional drug formulations (20-23).

The molecular formula of polyethylene glycol (PEG) is $\mathrm{H}(\mathrm{CH} 2 \mathrm{CH} 2 \mathrm{O}) \mathrm{nOH}$, it is one of medicinal synthetic polymer injections which can be used for humans approved by FDA. There are many advantages of PEG drugs such as: i) extending biologic half-life of drug, enhancing long-acting and sustained-release effect; ii) improving the solubility and stability of the drug; iii) reducing immunogenicity and antigenicity; iv) reducing enzyme degradation; v) enhancing the targeting function of drugs; vi) reducing the toxicity of some drugs. Based on the above advantages, we chose PEG to formulate polymeric nanoparticles because of its excellent biocompatibility and biodegradability, then we made a comparison between DANPs and PEG-DANPs to see their effect against NSCLC.

Aiming to develop a good nanoparticle carrier for DTX, we synthesized PEG-DANPs via the emulsion-evaporation cross-link method, and we carried out a series of experiments in vitro, the results demonstrated that PEG-DANPs were a promising modality for NSCLC.

\section{Materials and methods}

Materials. All reagents and solvents were used as received, without further purification. Monomethoxy polyethylene glycol with a molecular weight of $20,000 \mathrm{kDa}$ (mPEG 20,000), D,L-lactide, and stannous octoate were purchased from Sigma-Aldrich Chemical Corp. (Shanghai, China); DTX was purchased from Beijing Norzer Pharmaceutical Co., Ltd. (China, Beijing); free DTX (Aisu $\left.{ }^{\circledR}\right)$ was manufactured by Jiangsu Hengrui Medicine Co., Ltd. (Jiangsu, China); and 3-(4,5)-dimethyl-thiazol(-z-y1)-3,5-di-phenyltetrazolium bromide (MTT) was obtained from Amresco LLC (Solon, OH, USA); Annexin V-FITC apoptosis detection kit was purchased from 4A Biotech Co., Ltd (Beijing, China); VivoGlo ${ }^{\circledR}$ luciferin was purchased from Promega Corp. (Madison, WI, USA). Trypsin, fetal bovine serum (FBS) and RPMI-1640 medium were purchased from HyClone Laboratories (Logan, UT, USA) and culture flasks and dishes were from Corning Incorporated (Corning, NY, USA).

Cell line and animals. Human non-small lung cancer A549 cell line was provided by the Department of Pathology in the
Institute of Medicinal Biotechnology in Peking Union Medical College. A549 cells were cultured in RPMI-1640 medium supplemented with $10 \%$ heat inactivated FBS and incubated in a humidified atmosphere of $5 \% \mathrm{CO}_{2}$ and $95 \%$ air at $37^{\circ} \mathrm{C}$. Female BalB/c mice (6-8-week old) were used for antitumor efficacy studies and were purchased from Beijing Vital River Laboratories (Beijing, China).

Animals were acclimatized in the holding facility prior to the beginning of the study. All animal procedures were approved by the Institutional Animal Care and Use Committee of the Chinese Academy of Medical Sciences. All surgeries were performed under sodium pentobarbital anesthesia ( $5 \mathrm{mg} / \mathrm{ml}$ solution) and all efforts were made to minimize suffering. Lung tumor and other sections were routinely stained with hematoxylin and eosin (H\&E) and evaluated under a light microscope.

Preparation of DANPs and PEG-DANPs. DTX was dissolved in chloroform and ethanol to form solution A, albumin was dissolved in sterile water to form solution B. The solution A and B was mixed and stirred by homogenate machine for $5 \mathrm{~min}$ to form raw milk, which was high pressure homogenized, under 20,000 psi for 12 cycles. The chloroform of mixture was eliminated by using rotary evaporator for $25 \mathrm{~min}$ and followed by filtration through a $0.22-\mu \mathrm{m}$ filter.

DANPs and mPEG $(20,000 \mathrm{kDa})$ were added to the solution of boric acid buffer $(0.1 \mathrm{~mol} / 1, \mathrm{pH} 9.0)$ according to the ratio of 3:1 for stirring and the reaction was terminated via adding glycine $(1 \mathrm{mg} / \mathrm{ml}) 3 \mathrm{~h}$ later. Unbound HAS and PEG were removed by ultrafiltration (MWCO: $70 \mathrm{kDa})(24,25)$.

Determination via PAGE gel electrophoresis: DANPs and PEG-DANPs were determined via PAGE gel electrophoresis with iodine staining and mas blue staining.

Encapsulation efficiency and size distribution. Loading capacity was defined as the percentage of DTX by weight in the freeze-dried nanoparticles, and encapsulation efficiency was defined as the percentage of DTX by weight incorporated in the nanoparticles compared with the initial weight of DTX (26). Both were determined by high performance liquid chromatography (HPLC) (27). The content of DTX was assayed on an Agilent 1200LC HPLC system (Agilent Technologies, Santa Clara, CA, USA). The mobile phase consisted of a mixture of acetonitrile and water $(55: 45, \mathrm{v} / \mathrm{v})$ delivered at a flow rate of $0.5 \mathrm{ml} / \mathrm{min}$. The injection volume was $20 \mu \mathrm{l}$ and the wavelength was set at $230 \mathrm{~nm}$. The column temperature was $25^{\circ} \mathrm{C}$. The concentration of DTX was determined based on the peak area (standard curve as $\mathrm{y}=22.122 \mathrm{x}+45.3424, \mathrm{R}=0.9996$ ). Drug loading content $(\mathrm{DLC})=$ Content of physically loaded DANPs (PEG-DANPs)/Content of DANPs (PEG-DANPs) x $100 \%$. Encapsulation efficiency $(\mathrm{EE})=$ Content of physically loaded DANPs (PEG-DANPs)/Content of DANPs (PEG-DANPs) initially added $\mathrm{x} 100 \%$.

The mean diameter, size distribution and zeta potential of the DANPs and PEG-DANPs were measured by a Malven Nano ZS laser particle analyzer. All the analysis were performed three times $(28,29)$. The morphology of the DANPs and PEG-DANPs nanoparticles was analyzed by transmission electron microscopy (TEM). 
In vitro drug release. In vitro release of drugs were assayed using dialysis diffusion method $(30,31)$. Typically, $0.5 \mathrm{ml}$ of Aisu, DANPs and PEG-DANPs at DTX concentration of $1 \mathrm{mg} / \mathrm{ml}$ was sealed into a dialysis bag (MWCO $8000 \mathrm{kDa}$; Pierce). The bag was immersed in $40 \mathrm{ml} \mathrm{PBS} \mathrm{(140} \mathrm{mmol/l,}$ $\mathrm{pH} 7.4$ ) at $37^{\circ} \mathrm{C}$ with a shaking rate of $100 \mathrm{rpm}$ (27). At predetermined time-points, $0.2 \mathrm{ml}$ samples were taken from the bags and the same volume of fresh medium was added. Each sample was centrifuged at 10,000 rpm and the supernatant was assayed by HPLC.

Cell viability. The in vitro cytotoxic activity of DANPs and PEG-DANPs was evaluted by the MTT assay. Briefly, the A549 cells $\left(8 \times 10^{4}\right.$ cells $\left./ \mathrm{ml}\right)$ were seeded in 96 -well plates and incubated for $24 \mathrm{~h}$ to allow cell attachment. The cells were then treated with a series of PBS (control), Aisu, DANPs and PEG-DANPs, at $37^{\circ} \mathrm{C}(0.001,0.01,0.1,1,10$ and $100 \mu \mathrm{g} / \mathrm{ml})$. At the determined incubation time-points of $48 \mathrm{~h}, 20 \mu \mathrm{l}$ of MTT $(5 \mathrm{mg} / \mathrm{ml})$ was added and incubated for $4 \mathrm{~h}$, MTT was aspirated and $180 \mu \mathrm{l} /$ well of DMSO was added to dissolve the formazan crystals, and the plate was gently shaken for $10 \mathrm{~min}$. The optical density (OD) was measured at $490 \mathrm{~nm}$ by Synergy $\mathrm{H} 1 \mathrm{~m}$ monochromator-Based multi-mode microplate reader (Bio-Tek Instruments, Inc., Winooski, VT, USA). The cell inhibition was calculated according to the formula: Cell inhibition $(\%)=\left[1-\left(\mathrm{OD}_{\text {sample }}-\mathrm{OD}_{\text {blank }}\right) /\left(\mathrm{OD}_{\text {sample }}-\mathrm{OD}_{\text {blank }}\right)\right] \times 100 \%$. The results are expressed as means \pm SD of 3 measurements. No precipitation of DTX was found during the incubation procedure (32).

Cell apoptosis assay. Apoptotic cells were determined by dual staining with an Annexin $\mathrm{V}$ and propidium iodide (PI) kit (4A Biotech) according to the manufacturer's instructions (33). After $48 \mathrm{~h}$ of incubation in the exponential stage, A549 cells seeded in 12-well plates were treated for a further $48 \mathrm{~h}$ with $10 \mathrm{nmol} / \mathrm{ml}$ Aisu, DANPs and PEG-DANPs, respectively. After treatment, cells were washed twice with warm PBS, detached by trypsin without EDTA, then the following steps of collection, centrifuge, washing with warm PBS were performed, and further stained with PI and Annexin V-FITC for $15 \mathrm{~min}$ at room temperature in the dark. Apoptosis was then analyzed using a FACScan cytometer. Quadrant analysis was performed and cells that stained positive for both Annexin V-FITC and PI were designated as apoptotic, while unstained cells were designated as viable.

Cellular uptake of coumarin 6(C6)-loaded SPM (C6-SPM). For in vitro fluorescence imaging, the near-infrared fluorescent probe C6 was loaded into DANPs and PEG-DANPs to yield C6-SPM. Briefly, the polymers and excess C6 were co-dissolved in $\mathrm{CHCl}_{3}$ and a thin film was formed by the evaporation of $\mathrm{CHCl}_{3}$. $\mathrm{PBS}$ ( $\mathrm{pH} 7.4$ ) was added, followed by vortexing for $10 \mathrm{~min}$. The nanoparticles were extruded through a sterile membrane of pore size $220 \mathrm{~nm}$ (Millipore) to remove free C6. A549 cells in exponential-stage growth were incubated with C6-SPM at $37^{\circ} \mathrm{C}$ for $5,10,20$ and $30 \mathrm{~min}$, respectively, then the plate was rinsed three times with cold PBS and fixed with $4 \%$ paraformaldehyde for $10 \mathrm{~min}$. Finally, cells were observed by confocal laser scanning microscopy (CLSM, TCS SP2; Leica Microsystems GmbH, Wetzlar,
Germany). Images were examined using differential interference contrast and C6-SPM was recorded with the green channel (C6) with excitation at $488 \mathrm{~nm}$.

Pharmacokinetics. Twelve male Bal B/c mice were divided into three equal groups, and then injected with Aisu, DANPs and PEG-DANPs $(20 \mathrm{mg} / \mathrm{kg})$ through the tail vein. At $0.5,1,2,4,8,10,12$ and 24 h, following i.v. injection, $0.3 \mathrm{ml}$ blood from the carotid artery was collected into heparinized tubes at predetermined time-points. Blood was immediately centrifugated at $4,000 \mathrm{rpm}$ for $10 \mathrm{~min}$ to isolate the plasma, then $100 \mu \mathrm{l}$ of the plasma and $3 \mathrm{ml}$ of supernatant was mixed together and centrifuged at 3,000 rpm for $10 \mathrm{~min}$. Then organic phase was processed in a similar manner to the above, blow drying with nitrogen at $60^{\circ} \mathrm{C}$ water bath was carried out, and HPLC was used for the determination (34).

Hemolysis test. Fresh whole blood was collected from male guinea pigs. The blood was stirred quickly to take out fibrousprotein from the blood, $0.9 \%$ saline was added and centrifuged at 2,000 rpm for $15 \mathrm{~min}$. The blood was washed repeatedly until the supernatant did not turn red. The prepared mixture was made into $2 \%(\mathrm{v} / \mathrm{v})$ red cell suspensions. Aisu, DANPs and PEG-DANPs were diluted to different concentrations with $0.9 \%$ saline $(0.1,0.25,0.5$ and $0.75 \mathrm{mg} / \mathrm{ml})$. The same volume $(200 \mu \mathrm{l})$ of drug solution and red cell suspension was mixed at $37^{\circ} \mathrm{C}$ for $1 \mathrm{~h}$. The mixture was then centrifuged for $5 \mathrm{~min}$ at $2,000 \mathrm{rpm}$ s to separate the supernatant. Finally, the detection of the supernatant was at $576 \mathrm{~nm}$ with the formula: Hemolysis $(\%)=[(\mathrm{ABS}-\mathrm{ABS} 0) /(\mathrm{ABS} 100-\mathrm{ABS} 0)] \times 100 \%$. Here, ABS 100 and ABS 0 are the A values of the solution at 100 and $0 \%$ hemolysis, respectively. Results were reported as means \pm SD.

Tumor study and in vivo antitumor efficacy. All experimental procedures were performed in conformity with institutional guidelines and protocols for the care and use of laboratory animals. We chose $40 \mathrm{Bal} \mathrm{B/c}$ mice and randomly divided into four groups, respectively, the negative control group (glucose injection group), positive control group (Aisu group), DANPs group and PEG-DANPs group with 10 animals of each. The lung cancer A549 cells were suspended in BD Matrigel, and the mice in each group were subcutaneously implanted with $3 \times 10^{6}$ cells to establish the transplantation tumor model $(35,36)$. When the tumor volume reached $\sim 120 \mathrm{~mm}^{3}$, the mice were treated 4 times at 7-day intervals with $5 \%$ glucose injection (negative control), Aisu, DANPs or PEG-DANPs, respectively. All formulations were injected intravenously via the tail vein at a DTX dose of $20 \mathrm{mg} / \mathrm{kg}$. The body weight and tumor volume were measured simultaneously. Tumor volume was calculated using the equation of $\mathrm{V}=\mathrm{w}^{2} \mathrm{xl} / 2$. Here $\mathrm{w}$ and 1 are the width and length of the tumor. Forty-eight hours after the last treatment, the mice were sacrificed. The tumors with lung and the other major organs (including heart, liver, spleen and kidney) were removed, fixed in $10 \%$ formalin solution, and subjected to paraffin embedding for H\&E staining. 
Table I. Characterization of DANPs and PEG-DANPs.

\begin{tabular}{lcccr}
\hline & Average size $(\mathrm{nm})$ & Zeta potential $(\mathrm{mV})$ & DLC $(\%)$ & EE $(\%)$ \\
\hline DANPs & $163.4 \pm 3.76$ & $-19.4 \pm 0.18$ & $8.71 \pm 0.98$ & $93.58 \pm 0.86$ \\
PEG-DANPs & $169.19 \pm 2.36$ & $-18.2 \pm 0.21$ & $8.72 \pm 1.05$ & $95.4 \pm 5.50$ \\
\hline
\end{tabular}

DLC, drug loading content; EE, encapsulation efficiency.

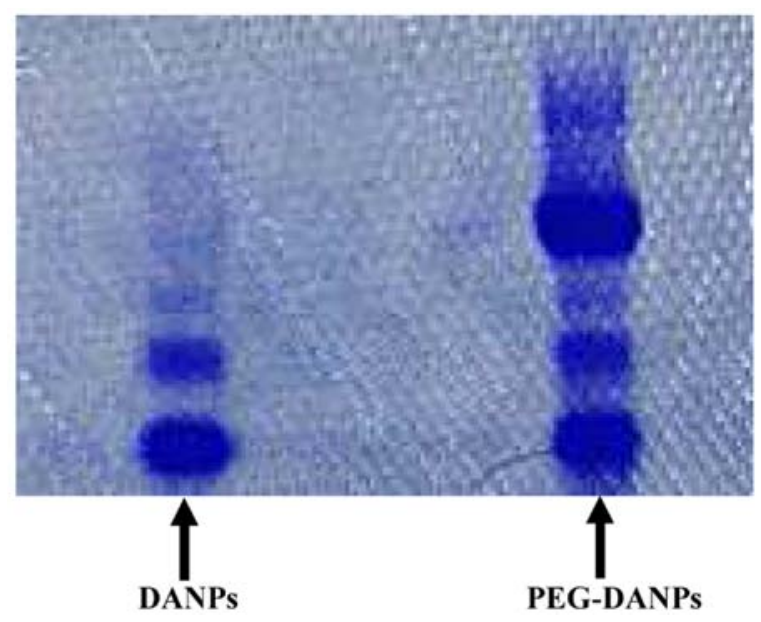

Figure 1. PAGE gel electrophoresis of DANPs and PEG-DANPs. PEGDANPs have two bands which are PEG and protein, respectively; DANPs have only one protein band.

Statistical analysis. Results are presented as means \pm SD. Statistical comparisons were made by t-test or ANOVA analysis. The level of significance was set at $\mathrm{P}<0.05$.

\section{Results}

Determination of DANPs and PEG-DANPs via PAGE gel electrophoresis. The PAGE gel electrophoresis shows that PEG-DANPs with two bands, PEG and protein, respectively; there is only one band in DANPs representing the protein (Fig. 1).

Characterization of nanoparticles. The characterization of DANPs and PEG-DANPs is presented in Table I. The DANPs had an average particle size of $163.4 \pm 3.76 \mathrm{~nm}$ (Fig. 2A), a zeta potential of $-19.4 \pm 0.18 \mathrm{mV}$, a polydispersity index of $0.14 \pm 0.03$; compared with the average particle size of PEG-DANPs of $169.19 \pm 2.36 \mathrm{~nm}$ (Fig. 2B), zeta potential $-18.2 \pm 0.21 \mathrm{mV}$, with a polydispersity index of $1.56 \pm 0.05$. The results of the size distribution indicated that the size of DANPs and PEG-DANPs was similar (37). The morphology images of TEM indicated that DANPs (Fig. 3A) and PEG-DANPs (Fig. 3B) were spherical with smooth surfaces. The drug loading of DANPs is $8.71 \pm 0.98 \%$, and an encapsulation efficiency $93.58 \pm 0.86 \%$, and PEG-DANPs is $8.72 \pm 1.05$ and $95.4 \pm 5.5 \%$, respectively.

In vitro drug release. Fig. 4 shows the release profile of Aisu, DANPs and PEG-DANPs. PEG-DANPs showed a slower and
A

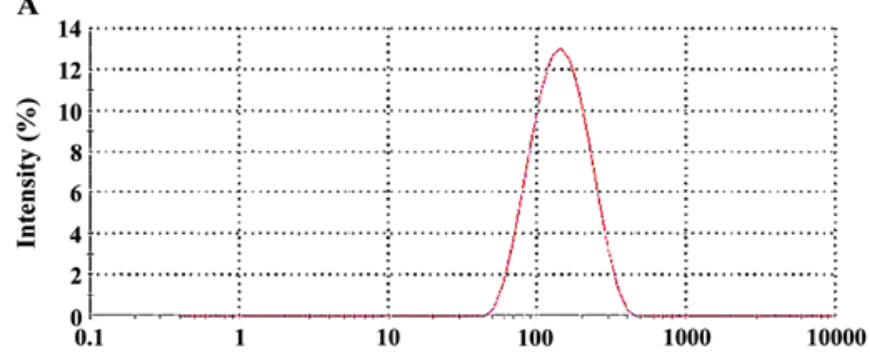

B

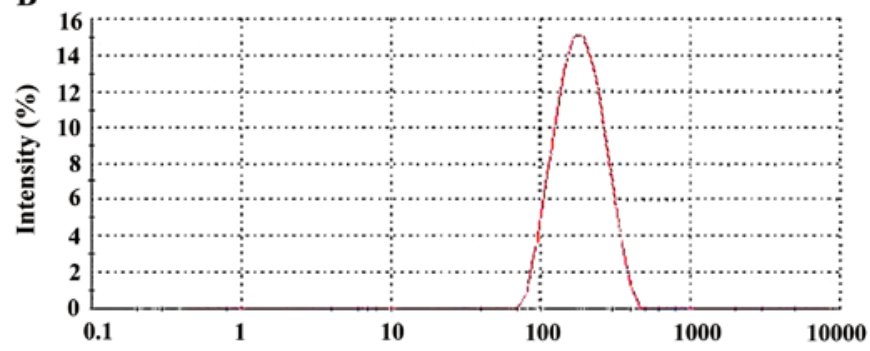

Figure 2. Particle size of DANPs (A) $(163.4 \pm 3.76 \mathrm{~nm})$ and PEG-DANPs (B $(169.19 \pm 2.36 \mathrm{~nm})$.

continuous release of the whole process compared to Aisu and DANPs. In this experiment, pH 7.4 PBS was selected to simulate the environment of blood. The release medium contained $0.5 \%$ of Tween- 80 in which the DTX was easily soluble.

Cell viability. Fig. 5 shows the result of the cytotoxicity of Aisu, DANPs and PEG-DANPs against A549 lung cancer cells. A549 cells were exposed to a series of equivalent concentrations of Aisu, DANPs and PEG-DANPs for $48 \mathrm{~h}$, and the inhibition rates were determined via the MTT method. The cell survival rate had a dose-dependent inverse relationship with the drug concentrations. PEG-DANPs accelerated cellular uptake of the drug and induced higher cytotoxicity in cancer cells than Aisu and DANPs, especially at lower DTX concentrations (0.001-0.1 $\mu \mathrm{g} / \mathrm{ml})$. However, A549 cells were more sensitive to Aisu than DANPs and PEG-DANPs at higher concentrations (1-100 $\mu \mathrm{g} / \mathrm{ml})$. Nanoparticles are internalized into cancer cells via endocytic mechanisms (38), while the free drug diffuses into cells according to the concentration gradient between the intracellular and extracellular environments. This is the reason why Aisu is more cytotoxic at higher concentrations.

PEG-DANPs increase DTX-induced apoptosis in A549 cells. DTX was described as an antimitotic agent which could 
A

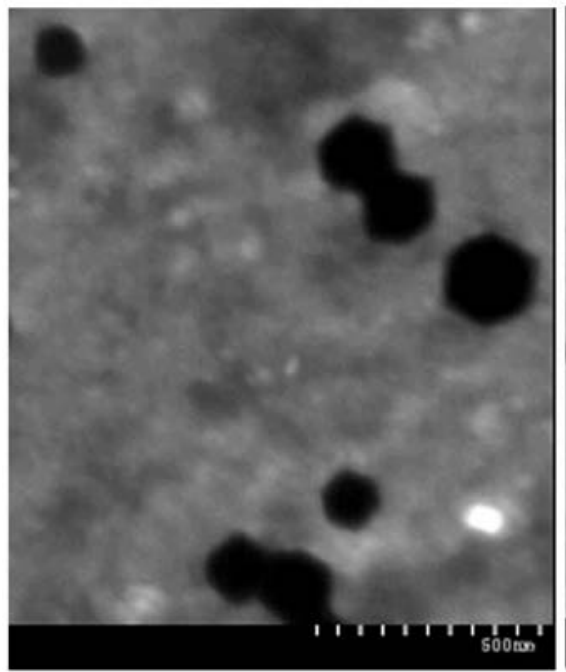

B

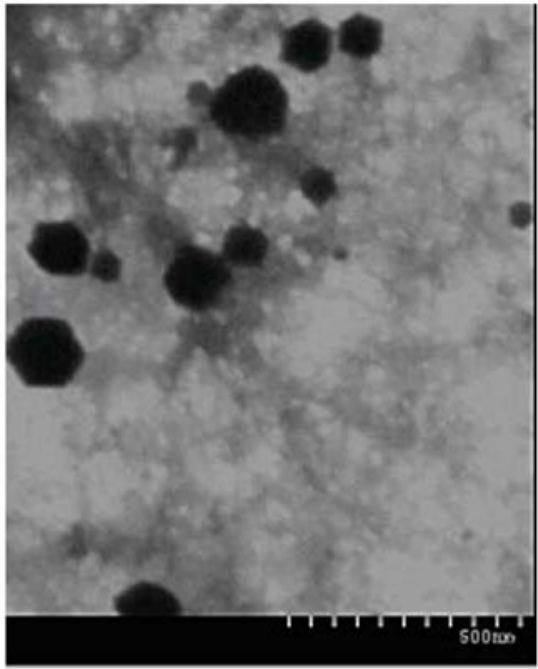

Figure 3. TEM scanning of blank DANPs (A) and PEG-DANPs (B) (x300,000).

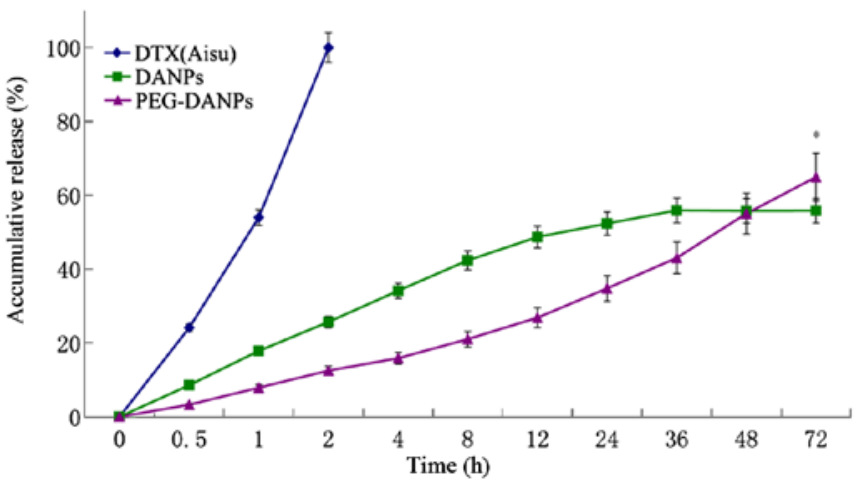

Figure 4. Accumulative DTX release from DANPs and PEG-DANPs in release medium $(0.5 \%$ of Tween- 80 in $\mathrm{PBS}, \mathrm{pH} 7.4)$ at $(37 \pm 0.5 \mathrm{C}(\mathrm{n}=3)$.

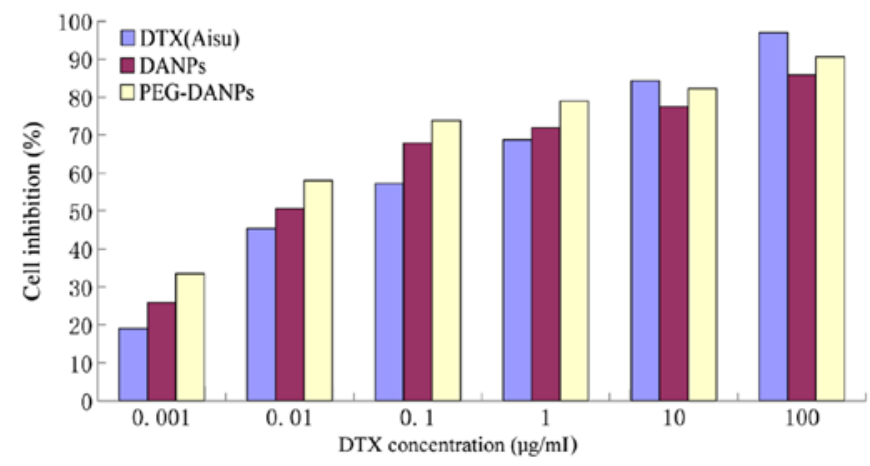

Figure 5. Inhibition of A549 lung cancer cells cultured with Aisu, DANPs and PEG-DANPs after $48 \mathrm{~h}$ at the same dose. All the data are presented as means \pm SD $(n=3)$. The PEG-DANPs induced higher cytotoxicity in cancer cells than Aisu and DANP especially at lower DTX concentrations (0.001-0.1 $\mu \mathrm{g} / \mathrm{ml})$.

bind to $\beta$-tubulin, resulting in block of the cell cycle at the G2/M phase and apoptosis of cells $(39,40)$. According to a previous study (41), encapsulation of DTX in nanoparticles further increased apoptosis of prostate cancer cells. Given that PEG-DTX-HANPs demonstrated stronger in vitro cytotox-
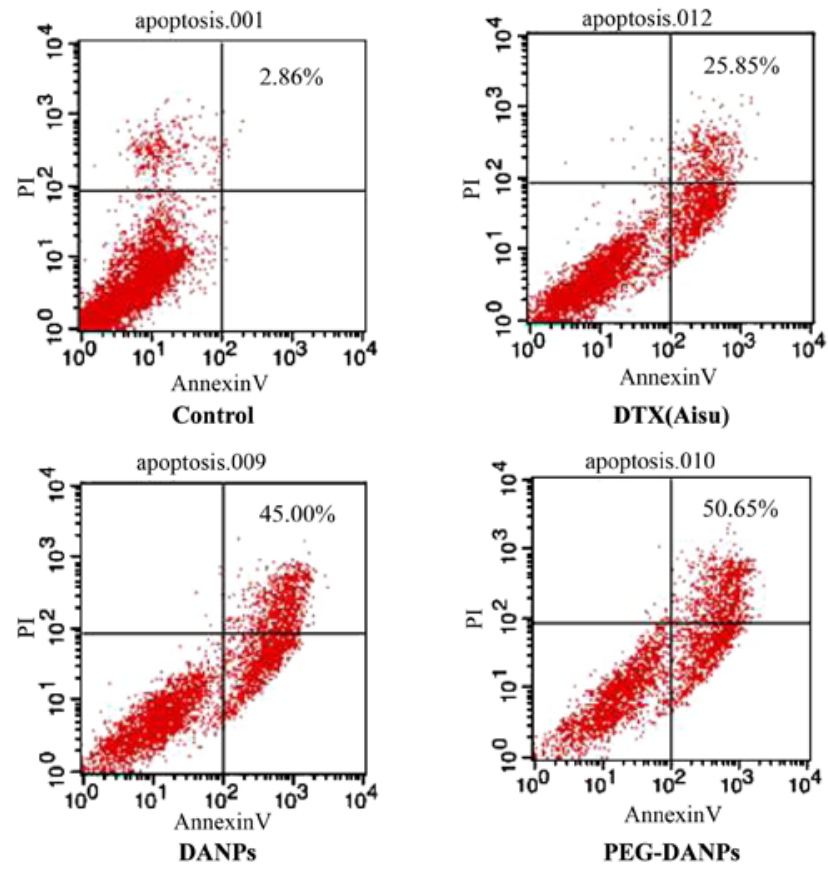

Figure 6. Flow cytometry detected cell apoptosis in A549 cells incubated for $48 \mathrm{~h}$ with $10 \mathrm{nmol} / \mathrm{ml}$ negative controls, Aisu, DANPs and PEG-DANPs, respectively.

icity than DANPs and Aisu, we performed apoptosis assays using Annexin V-FITC and PI staining to compare induction of apoptosis. As predicted, PEG-DANPs (55.65\%) increased late apoptosis in A549 cells compared with DANPs and Aisu (25.85 and 43.00\%) (Fig. 6).

The comparison of in vitro cellular uptake. Cellular uptake is very important in the nanodrug delivery system. Poor cellular uptake may result in low levels of intracellular DTX, ultimately leading to unsatisfactory therapeutic effects. Cellular uptake of C6-SPM was qualitatively visualized by CLSM and the internalization speed was roughly estimated. The CLSM images of A549 cells after incubation with C6-SPM for 5, 10, 

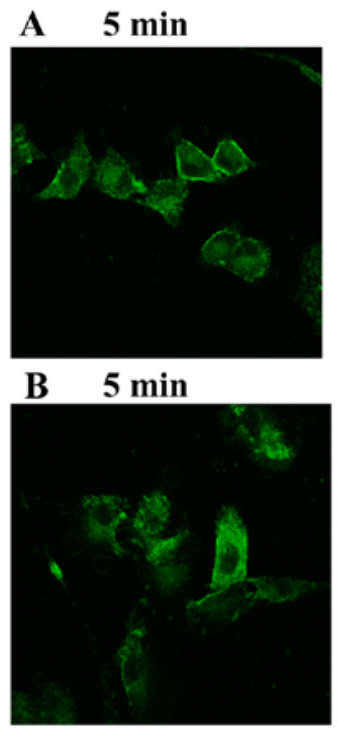

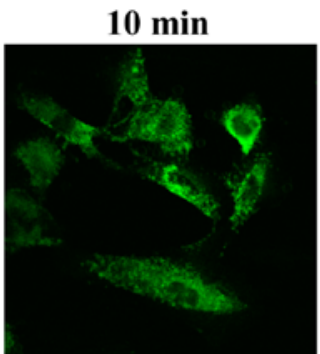

$10 \mathrm{~min}$

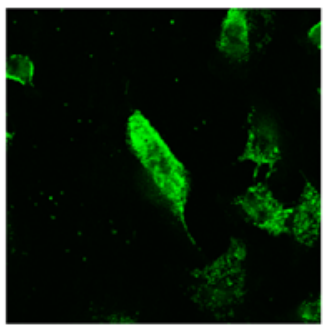

$20 \mathrm{~min}$

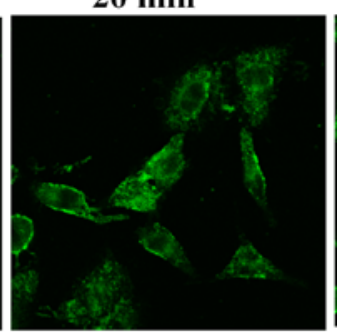

$20 \mathrm{~min}$

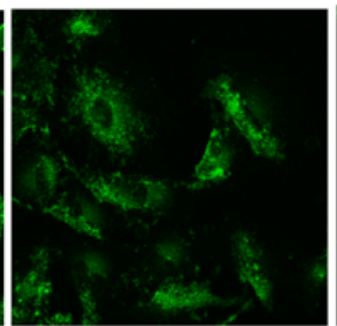

$30 \mathrm{~min}$

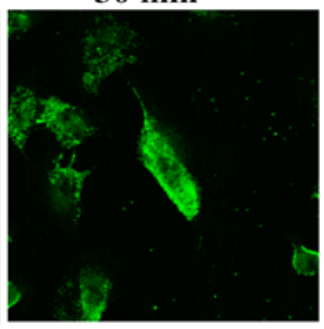

$30 \mathrm{~min}$

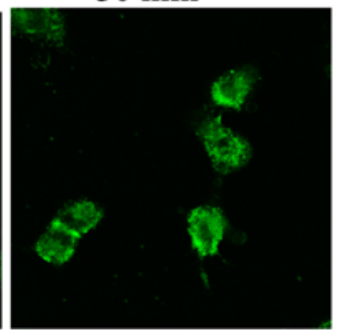

Figure 7. CLSM images of A549 cells incubated with DANPs (A) and PEG-DANPs (B) for 5, 10, 20 and 30 min, respectively. (Scale bar, 37.5 m).

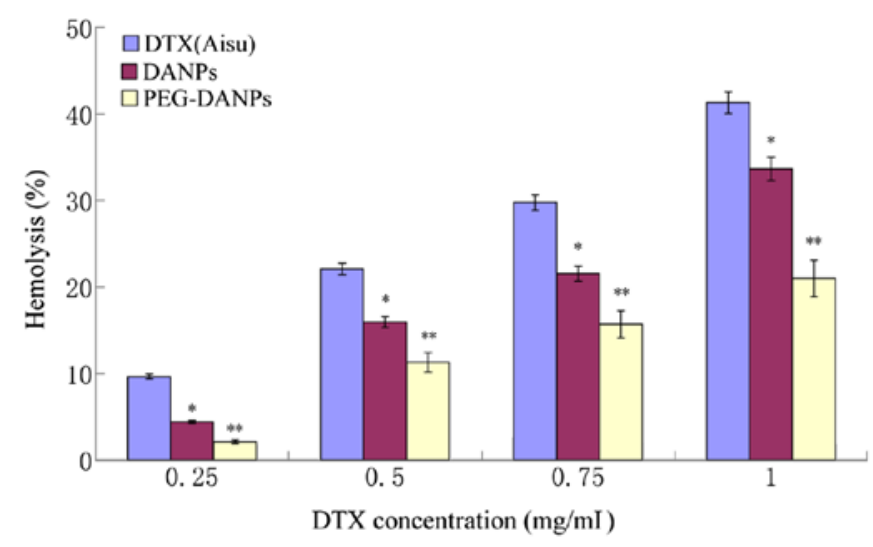

Figure 8. Hemolysis test of Aisu, DANPs and PEG-DANPs at different concentrations, the hemolysis levels in PEG-DANPs group were significantly lower than those in Tween-80 and Duopafei ${ }^{\circledR}$ groups $\left({ }^{*} \mathrm{P}<0.05\right)$.

20 and $30 \mathrm{~min}$ are shown in Fig. 7A. CLSM images at 5 and 10 min showed that C6-SPM fluorescence (green) was closely located around the membrane, indicating that C6-SPM was not internalized into A549 cells. However, when the incubation time was extended to $20 \mathrm{~min}, \mathrm{C6}$-SPM was successfully internalized into A549 cells. Nanoparticles have previously been reported to be internalized into the cytoplasm together with the entrapped drug via an endocytic mechanism (42). The process demonstrated in Fig. 7B indicated that PEG-DANPs could be absorbed sooner and easier into the nucleus than DANPs.

Pharmacokinetics of DANPs and PEG-DANPs. The compartment model was simulated using the WinNonlin 4.0 program and the parameters of pharmacokinetics were obtained $(29,43)$. In the present study, after the injection of DANPs and PEG-DANPs, the DTX concentration was still measurable after $24 \mathrm{~h}$, the concentration of PEG-DANPs is higher than DANPs. On the contrary, the Aisu was not detectable even after $12 \mathrm{~h}$. The difference in the DTX concentration between

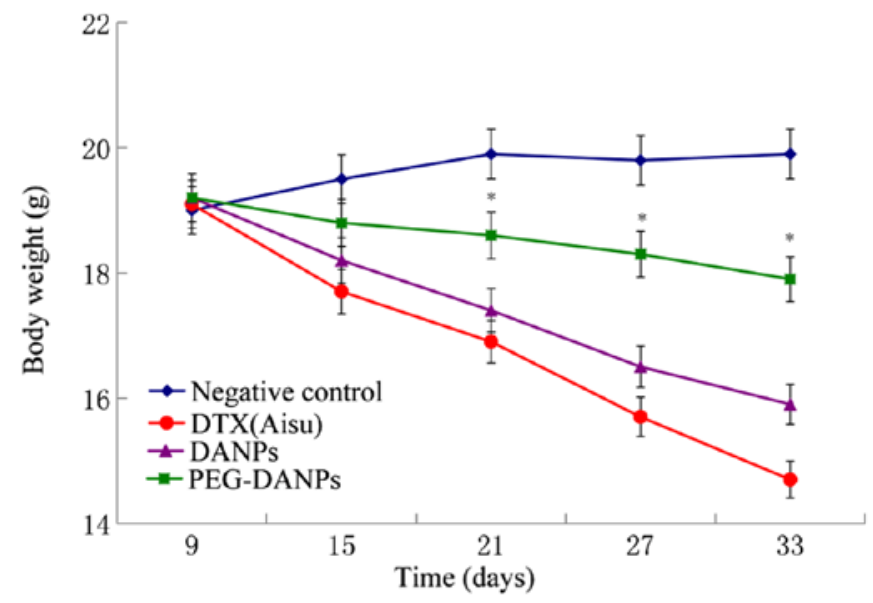

Figure 9. Changes in the body weight of A549 tumor-bearing nude mice in the Aisu, DANPs and PEG-DANPs (male, $n=10$ ). The drugs were intravenously administered 4 times at 3-day intervals at a dose of $20 \mathrm{mg} / \mathrm{kg}$. The body weights of the Aisu group were significantly lighter than those of the PEG-DANPs group ( $\mathrm{P}<0.01)$.

the three drugs was that the nanoparticles were generally rather present in blood in PEG-DANPs group than in DANPs and Aisu group. This is probably due to the slower distribution of DTX from nanoparticles into tissues because of its stability and sustained release. The pharmacokinetic parameters of the Aisu, DANPs and PEG-DANPs group are shown in Table II. The PEG-DANPs significantly enhanced the half-life of DTX.

Hemolysis test. All the injections and other potential immunohemolysis-causing or non-immunohemolysis-causing pharmaceutic preparation need to be tested prior to use. Thus, we made the exiperiments to test whether DANPs and PEG-DANPs are able to cause the breakdown or destruction of red blood cells and red cell aggregation. In addition, it can reflect the extent of the breakdown of RBC wall caused by chemical agents during the injection. In Fig. 8, the results of the hemolysis test as the hemolysis percent vs. DTX concen- 

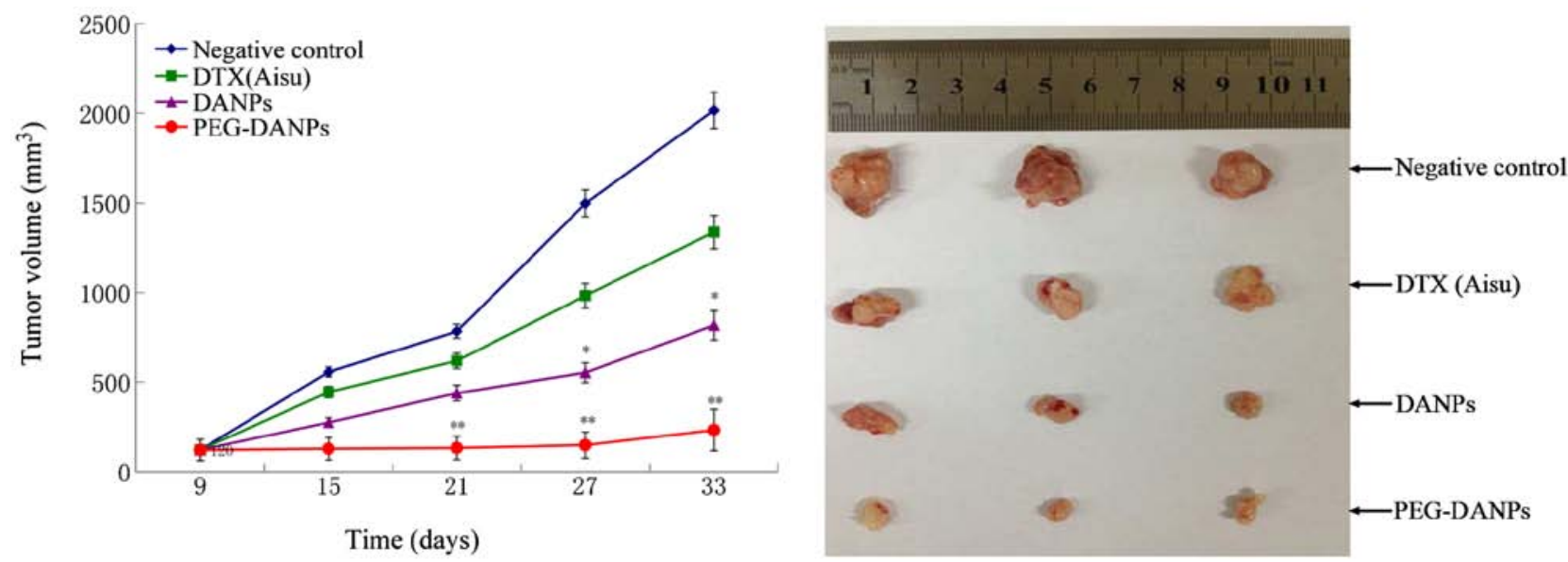

Figure 10. Changes in the tumor volume of A549 tumor-bearing nude mice of the Aisu, DANPs and PEG-DANPs (male, $\mathrm{n}=10$ ). The drugs were intravenously administered 4 times at three-day intervals at a dose of $20 \mathrm{mg} / \mathrm{kg}$. The tumor volume of the DANPs group and PEG-DANPs group were much smaller than those of the negative control group, the latter is more significant $\left({ }^{*} \mathrm{P}<0.05,{ }^{* *} \mathrm{P}<0.01\right)$.

Table II. Pharmacokinetic parameters of three drugs $(n=4$, means $\pm \mathrm{SD})$

\begin{tabular}{lccc}
\hline Parameters & Aisu $^{\circledR}$ & DANPs & PEG-DANPs \\
\hline T 1/2 /h & $2.13 \pm 0.16$ & $3.13 \pm 0.13$ & $6.58 \pm 0.18^{\mathrm{a}, \mathrm{b}}$ \\
AUC/mg.h.L-1 & $6.50 \pm 0.66$ & $7.15 \pm 0.52$ & $11.30 \pm 0.85^{\mathrm{a}, \mathrm{b}}$ \\
Cl/mL.h-1 & $2.61 \pm 0.15$ & $2.38 \pm 0.6$ & $1.38 \pm 0.09^{\mathrm{a}, \mathrm{b}}$ \\
\hline
\end{tabular}

${ }^{\mathrm{a}} \mathrm{P}<0.05$, compared with DANPs; ${ }^{\mathrm{P}} \mathrm{P}<0.01$, compared with Aisu.

tration are presented. Aisu displayed much more toxicity toward red blood cells (RBC) than DANPs and PEG-DANPs at the same concentration $(\mathrm{P}<0.05)$. The results indicated that PEG-DANPs caused the lowest hemolysis between the three drugs.

Anticancer effects of PEG-DANPs in vivo. Tumor-bearing nude mice were injected with Aisu, DANPs and PEG-DANPs, and their therapeutic effects were examined by measuring the suppression of body weight and tumor growth. The body weight of the mice in the negative control groups was basically unchanged or slightly increased, while the mice in the drug groups all lost weight in the process of the treatment. However, the reduction range in the Aisu groups was more significant than the other two groups $(\mathrm{P}<0.01)$ (Fig. 9), suggesting severe systemic toxicity in addition to tumor toxicity. It was found that all the tumor volumes treated with Aisu, DANPs and PEG-DANPs were much smaller than those of negative control groups treated with the same dose $(\mathrm{P}<0.05)$, and the tumors of PEG-DANPs groups were obviously smaller than those of Aisu groups (Fig. 10), indicating that PEG-DANPs are the most effective in inhibiting tumor growth among the three.

\section{Discussion}

In the present study, zeta potential of DANPs and PEG-DANPs was $-19.4 \pm 0.18$ and $-18.2 \pm 0.21 \mathrm{mV}$, respectively, which indicated that they had a negative surface charge. Our experiments indicated that the DANPs presented high drug loading and encapsulation efficiency of $8.71 \pm 0.98$ and $93.58 \pm 0.86 \%$; for PEG-DANPs, the values are $8.72 \pm 1.05$ and $95.4 \pm 5.5 \%$. In vitro release, compared with Aisu, DANPs and PEG-DANPs presented a more sustained manner of release, this is because DTX is encapsulated in the core portion, it has to go through the process of diffusion before release which lead to the delayed effect. In the present study, the gradual degradation of the copolymer and diffusion of drug are presented for the release of the loaded drug, this is the same as previous research results (44). Furthermore, Fig. 3 indicates that PEG-DANPs are superior to DANPs in vitro drug release, this is due to the PEG which in the surface of the nanoparticles. PEG-DANPs could be also used as a platform for the incorporation of active targeting moiety (39). PEG-DANPs could not only minimize the exposure of normal tissues but also increased the accumulation of the therapeutic drug in the tumor site compared to Aisu (43), all the characteristics show its potential applicability as a drug delivery system. The results of the pharmacokinetics indicated that PEG-DANPs had the longest circulation time, which was the same as in in vitro sustained release of DTX from the nanoparticles. As a result, the enhanced half-life of the PEG-DANPs was due to its longer circulation effect and residence time in vivo. In addition, the incorporation of DTX in nanoparticles produced better drug solubilization than that in Tween-80 in vitro release test. The PEG chain of PEG-DANPs is hydrophilic, it is currently thought to act as a protector to achieve long circulation time of drugs in the blood. It was able to accumulate in liver, spleen and lung, and was finally released from these organs to blood circulation according to the drug concentration gradient (40), which resulted in a sustained blood level compared to Aisu and DANPs. From that discussed above, we can make a conclusion that PEG-DANPs can not only increase the concentration and uptake of antitumor drugs in the tumors but also prolong the time that drugs are sustained in the blood $(45,46)$, all of which are the main approaches to increase antitumor activity and inhibit tumor growth in chemotherapy.

PEG-DANPs with an appropriate particle size can significantly accumulate in the tumor via the EPR effect, 
this is called size-dependent passive targeting. The size of PEG-DANPs is $\sim 169 \mathrm{~nm}$, it can preferential accumulate and stay in tumor tissues compared to normal tissues. Moreover, from the pharmacokinetic test, we concluded that the DTX concentration in blood of the PEG-DANPs group was higher than that of DANPs and Aisu group. These points may well explain why PEG-DANPs delayed tumor development significantly better than the two other drugs. Fig. 8, shows the body weight changes in the tested mouse groups. The body weight loss of the mice in Aisu groups was significantly more than the DANPs and PEG-DANPs groups, especially the PEG-DANPs group. These results show that PEG-DANPs has less toxicity to normal organs and less systemic toxicity.

In summary, in our experiments the PEG-DANPs were fabricated by emulsion-evaporation cross-link method against NSCLC. The in vitro release and in vivo pharmacokinetics studies showed a sustained and continuous release effect; the in vitro cytotoxicity study proved the dose- and time-dependent manner against A549 lung cancer cells; the cellular uptake test in vitro demonstrated that PEG-DANPs could be absorbed faster and easier into the nucleus; the hemolysis test indicated PEG-DANPs had the lowest side-effects compared to Aisu and DANPs; the in vivo evaluation pointed to PEG-DANPs having superior antitumor effect and relatively lower sideeffects compared with Aisu and DANPs. PEG-DANPs exerted promising therapeutic effects on NSCLC, and it is a good drug-delivery platform for the treatment of NSCLC.

\section{References}

1. Gridelli C, Maione P, Comunale D and Rossi A: Adjuvant chemotherapy in elderly patients with non-small cell lung cancer. Cancer Contr 14: 57-62, 2007.

2. Wu SH: Effect of docetaxel with cisplatin in treating advanced non-small cell lung cancer. J Hainan Medical College 16: 1615-1617, 2010 (In Chinese).

3. Li XT: Docetaxel and cisplatin treatment of non-small cell lung cancer analysis. Chin J Modern Drug Appl 4: 34-35, 2010.

4. Lu XM and Mao GX: A study of docetaxel plus cisplatin versus gemcitabine plus cisplatin in treating advanced non-small cell lung cancer. J Basic Clin Oncol 22: 308-310, 2009.

5. Xu Y: Docetaxel combined with cisplatin in the treatment of advanced non-small cell lung cancer. Chin J Modern Drug Applic 4: 26-28, 2010.

6. Shao JH: Efficacy of docetaxel combined with nedaplatin or cisplatin in patients with advanced non-small cell lung cancer Chin J New Drugs 19: 599-601, 2010.

7. Zhang CH, Ren ZH, Li M, et al: Cisplatin plus docetaxel combination in the first-line treatment of advanced non-small cell lung cancer. Chin J Clin Oncol Rehab 17: 54-56, 2010.

8. Stineheombe TE and Socinski MA: Considerations for secondline therapy of non-small cell lung cancer. Oncologist 13 (Suppl 1): 28-36, 2008.

9. Xu YZ and Xu LW: The exploration of 70 years of age or older chemotherapy against non-small cell lung cancer cells. Zhejiang Clin Med 9: 1226-1227, 2007 (In Chinese).

10. Li JJ: A study of cisplatin plus docetaxel or gemcitabine in treating advanced non small cell lung cancer. Mod Med 18: 54-55, 58, 2011.

11. Xiong Y, Zhou TC, Liu Y, Wang ZW, Lin XL, Song XL, Shi XY and Liao ZW: Clinical analysis of efficacy in docetaxel plus cisplatin chemotherapy with 3-DCRT treating the patients with locally advanced NSCLC. Chin J Cancer Prev Treat 17: 699-702, 2010.

12. Greco FA, Spigel DR, Burris HA III, Shipley DL, Farley C, Gandhi J, Houston GA and Hainsworth JD: Weekly docetaxel versus docetaxel/gemcitabine in elderly/poor performance status (PS) patients (pts) with stage III B/ IV non-small cell lung cancer (NSCLC): Randomized phase III trial of the Minnie Pearl Cancer Research Network. J Clin Oncol 25 (Suppl): 7534, 2007.
13. Kudoh S, Takeda K, Nakagawa K, Takada M, Katakami N, Matsui K, Shinkai T, Sawa T, Goto I, Semba H, et al: Randomized phase study of docetaxel versus vinorelbine for elderly patients with advanced non-small cell lung cancer (NSCLC): Results of a west Japan thoracic oncology group trial(WJTOG 9904). J Clin Oncol 24: 3657-3663, 2006.

14. Singer EA and Srinivasan R: Intravenous therapies for castration-resistant prostate cancer: Toxicities and adverse events. Urol Oncol 30 (Suppl 4): S15-S19, 2012.

15. Jin MJ, Piao SJ, Jin TX, Jin ZH, Yin XZ and Gao ZG: Improved anti-tumor efficiency against prostate cancer by docetaxel-loaded PEG-PCL micelles. J Huazhong Univ Sci Technolog Med Sci 34: 66-75 2014.

16. Yared JA and Tkaczuk KH: Update on taxane development: New analogs and new formulations. Drug Des Devel Ther 6: 371-384, 2012.

17. Liu Q, Li R, Zhu Z, Qian X, Guan W, Yu L, Yang M, Jiang X and Liu B: Enhanced antitumor efficacy, biodistribution and penetration of docetaxel-loaded biodegradable nanoparticles. Int J Pharm 430: 350-358, 2012.

18. Ghosh SC, Neslihan Alpay S and Klostergaard J: CD44: A validated target for improved delivery of cancer therapeutics. Expert Opin Ther Targets 16: 635-650, 2012.

19. Gao W, Xiang B, Meng TT, Liu F and Qi XR: Chemotherapeutic drug delivery to cancer cells using a combination of folate targeting and tumor microenvironment-sensitive polypeptides. Biomaterials 34: 4137-4149, 2013.

20. Wang F, Zhang D, Zhang Q, Chen Y, Zheng D, Hao L, Duan C, Jia L, Liu G and Liu Y: Synergistic effect of folate-mediated targeting and verapamil-mediated P-gp inhibition with paclitaxel-polymer micelles to overcome multi-drug resistance. Biomaterials 32: 9444-9456, 2011.

21. Ganesh S, Iyer AK, Gattacceca F, Morrissey DV and Amiji MM: In vivo biodistribution of siRNA and cisplatin administered using CD44-targeted hyaluronic acid nanoparticles. J Control Release 172: 699-706, 2013.

22. Iyer AK, Greish K, Seki T, Okazaki S, Fang J, Takeshita K and Maeda $\mathrm{H}$ : Polymeric micelles of zinc protoporphyrin for tumor targeted delivery based on EPR effect and singlet oxygen generation. J Drug Target 15: 496-506, 2007.

23. Fang J, Nakamura $\mathrm{H}$ and Maeda $\mathrm{H}$ : The EPR effect: Unique features of tumor blood vessels for drug delivery, factors involved, and limitations and augmentation of the effect. Adv Drug Deliv Rev 63: 136-151, 2011.

24. Tang N, Du G, Wang N, Liu C, Hang H and Liang W: Improving penetration in tumors with nano assemblies of phospholipids and doxorubicin. J Natl Cancer Inst 99: 1004-1015, 2007.

25. Li RY: Docetaxel-loaded PEG-albumin nanoparticles for antimetastasis in murine 4T1 breast cancer. Yanbian University Ph.D. Thesis, 2012.

26. Li YF, Li PR, Jin MJ, Jiang C and Gao Z: Docetaxel-encapsulating small-sized polymeric micelles with higher permeability and its efficacy on the orthotopic transplantation model of pancreatic ductal adenocarcinoma. Int J Mol Sci 15: 23571-23588, 2014.

27. Li Y, Zhao JP, Wang YN, Fu ZP, Guo N, Dong JR and Xia Y: Experimental study of serum containing juglone anti-tumor in vitro. Trans Beijing Inst Technol 33: 545-550, 2013.

28. Zheng D, Li X, Xu H, Lu X, Hu Y and Fan W: Study on docetaxelloaded nanoparticles with high antitumor efficacy against malignant melanoma. Acta Biochim Biophys Sin (Shanghai) 41: 578-587, 2009.

29. Yuan Z, Chen D, Zhang S and Zheng Z: Preparation, characterization and evaluation of docetaxel-loaded, folate-conjugated PEG-liposomes. Yakugaku Zasshi 130: 1353-1359, 2010.

30. Gao Y, Yu HR and Wang HX: Preparation of paclitaxel-loaded nanoparticles by albumin fragments. J Chongqing Med Univ 37: 795-798, 2012.

31. Shi NQ, Gao W, Xiang B and Qi XR: Enhancing cellular uptake of activable cell-penetrating peptide-doxorubicin conjugate by enzymatic cleavage. Int J Nanomed 7: 1613-1621, 2012.

32. Li JQ, Yang ZZ, Meng TT and Qi XR: The use of cationic liposomes to co-deliver docetaxel and siRNA for targeted therapy of hepatocelluar carcinoma. J Chin Pharm Sci 23: 667-673, 2014

33. Li YF, Jin MJ, Shao S, Huang W, Yang F, Chen W, Zhang S, Xia G and Gao Z: Small-sized polymeric micelles incorporating docetaxel suppress distant metastases in the clinically-relevant 4T1 mouse breast cancer model. BMC Cancer 14: 329-344, 2014. 
34. Chen W, Gu B, Wang H, Pan J, Lu W and Hou H: Development and evaluation of novel itraconazole-loaded intravenous nanoparticles. Int J Pharm 362: 133-140, 2008.

35. Kim D, Gao ZG, Lee ES and Bae YH: In vivo evaluation of doxorubicin-loaded polymeric micelles targeting folate receptors and early endosomal $\mathrm{pH}$ in drug-resistant ovarian cancer. Mol Pharm 6: 1353-1362, 2009.

36. Gao ZG, Fain HD and Rapoport N: Controlled and targeted tumor chemotherapy by micellar-encapsulated drug and ultrasound. J Control Release 102: 203-222, 2005.

37. Zhang L, Yang M, Wang Q, Li Y, Guo R, Jiang X, Yang C and Liu B: 10-Hydroxy camptothecin loaded nanoparticles: Preparation and antitumor activity in mice. J Control Release 119: 153-162, 2007.

38. Xiao L, Xiong X, Sun X, Zhu Y, Yang H, Chen H, Gan L, Xu H and Yang X: Role of cellular uptake in the reversal of multidrug resistance by PEG-b-PLA polymeric micelles. Biomaterials 32 : $5148-5157,2011$.

39. Chen W, Zhan C, Gu B, Meng Q, Wang H, Lu W and Hou H: Targeted brain delivery of itraconazole via RVG29 anchored nanoparticles. J Drug Target 19: 228-234, 2011.

40. Mu L, Teo MM, Ning HZ, Tan CS and Feng S: Novel powder formulations for controlled delivery of poorly soluble anticancer drug: Application and investigation of TPGS and PEG in spraydried particulate system. J Control Release 103: 565-575, 2005.
41. Li X, Li R, Qian X, Ding Y, Tu Y, Guo R, Hu Y, Jiang X, Guo W and Liu B: Superior antitumor efficiency of cisplatin-loaded nanoparticles by intratumoral delivery with decreased tumor metabolism rate. Eur J Pharm Biopharm 70: 726-734, 2008.

42. Danhier F, Lecouturier N, Vroman B, Jérôme C, MarchandBrynaert J, Feron O and Préat V: Paclitaxel-loaded PEGylated PLGA-based nanoparticles: In vitro and in vivo evaluation. J Control Release 133: 11-17, 2009.

43. Gong C, Xie Y, Wu Q, Wang Y, Deng S, Xiong D, Liu L, Xiang M, Qian Z and Wei Y: Improving anti-tumor activity with polymeric micelles entrapping paclitaxel in pulmonary carcinoma. Nanoscale 4: 6004-6017, 2012.

44. Van Vlerken LE, Vyas TK and Amiji MM: Poly(ethyleneglycol)modified nanocarriers for tumor-targeted and intracellular delivery. Pharm Res 24: 1405-1414, 2007.

45. Wang F, Zhang D, Zhang Q, Guo S, Zheng D, Hao L, Guo H and Li C: Tissue distribution and pharmacokinetics evaluation of DOMC-FA micelles for intravenous delivery of PTX. J Drug Target 21: 137-145, 2013.

46. Wang J, Huo MR and Zhang XY: Progress in hyaluronic acidbased targeted nano-drug delivery systems. Chin J Pharmaceut 44: 828-835, 2013 